\title{
EDITORIAL
}

\section{LIPOTOXICIDAD EN MÚSCULO ESQUELÉTICO, UN CAMINO PARA INVESTIGAR}

\author{
SKELETAL MUSCLE LIPOTOXICITY, A WAY TO INVESTIGATE
}

Para quienes leen un editorial de la Revista de la Sociedad Argentina de Diabetes saben que la diabetes mellitus tipo 2 (DM2) es una enfermedad compleja, generada a partir de múltiples procesos fisiopatológicos que aún atraen debate. Lo que se encuentra fuera de discusión, a esta altura del conocimiento, es la teoría acerca de que la "grasa ectópica", acumulada en células distintas de los adipocitos, conduce a resistencia a la insulina órganoespecífica conocida como lipotoxicidad'.

Ante situaciones de obesidad e insulinorresistencia, la capacidad deteriorada o sobrepasada de los adipocitos subcutáneos de almacenar ácidos grasos bajo la forma de di o triglicéridos conlleva a un aumento del depósito de grasa visceral y también al depósito ectópico de lípidos en otros órganos $^{2}$. Dentro de los lugares donde más frecuentemente se identifica esta acumulación ectópica se encuentra el hígado, donde puede originarse la enfermedad hepática grasa no alcohólica (EHGNA), en el páncreas, corazón y músculo esquelético.

El depósito ectópico de ácidos grasos en el músculo esquelético es especialmente importante en el control de la normoglucemia, dado que este tejido es responsable de la utilización del 30\% de la glucosa basal, y entre el 70 y el $90 \%$ en respuesta a la insulina ${ }^{3}$. Por lo tanto, procesos que alteren el consumo de glucosa por el músculo contribuyen al desarrollo de insulinorresistencia y diabetes. De hecho la capacidad de los ácidos grasos libres para alterar el metabolismo de la glucosa en el músculo se describió hace más de 50 años ${ }^{4}$.

En situaciones de sobrepeso y obesidad es frecuente encontrar mayor disponibilidad de lípidos circulantes provenientes en parte de la lipólisis aumentada en el tejido adiposo, el cual suele estar inflamado en estas situaciones ${ }^{5}$, y en parte de la síntesis hepática de novo. Esta mayor disponibilidad de ácidos grasos contribuye, en primera instancia, al aumento de síntesis hepática de lipoproteínas ricas en triglicéridos como VLDL (very low density lipoprotein, en inglés). A su vez, el catabolismo ralentizado de estas lipoproteínas en individuos con sobrepeso/obesidad e insulinorresistencia ${ }^{6}$ determina su mayor permanencia en plasma y la formación de remanentes lipoproteicos con mayor carácter aterogénico. El aumento en estas lipoproteínas circulantes, así como la presencia de ácidos grasos libres unidos a la albúmina, contribuyen a un incremento en la captación de los mismos por parte del músculo ${ }^{7}$ y alteraciones en su transporte, oxidación y esterificación ${ }^{8}$. El desbalance entre el ingreso de ácidos grasos y su oxidación en los miocitos tendrían un rol protagónico en la lipotoxicidad. Asimismo el acrecentamiento en la concentración plasmática de ácidos grasos libres altera la señalización de la insulina en este tejido, y promueve insulinorresistencia y aumento intramiocelular de lípidos.

Sin embargo es la acumulación de metabolitos tóxicos derivados de la oxidación incompleta de los ácidos grasos -como acilcarnitina, ceramidas y diacilglicéridos, entre otros- la principal responsable de la insulinorresistencia muscular. Las ceramidas, que son sintetizadas de novo a partir de ácidos grasos como el palmítico, demostraron desarrollar un rol crucial en el deterioro de la sensibilidad a la insulina en las células musculares ${ }^{9}$.

El estudio de los mecanismos oxidativos de los ácidos grasos en el músculo y la dilucidación de distintas vías enzimáticas involucradas son fundamentales considerando que este tejido es un importante target para el estudio de la fisiopatología de la insulinorresistencia, así como para la búsqueda de alternativas terapéuticas. En este sentido, el trabajo presentado en esta edición: "Lipotoxicidad en músculo esquelético y su relación con la resistencia insulínica. Estudios en un modelo experimental de síndrome metabólico" investiga mecanismos involucrados en la lipotoxicidad del músculo esquelético en un modelo animal de insulinorresistencia, 
y se centra en el estudio de las enzimas carnitina palmitoil transferasa muscular (M-CPT), factores de transcripción que controlan los procesos como la captación, transporte, oxidación y esterificación de ácidos grasos como el receptor activado por proliferadores peroxisomales $\alpha$ (PPAR $\alpha$ ), y la proteína kinasa activada por AMP (AMPK), reguladora de eventos fisiológicos, incluidos el crecimiento y proliferación celular, la función y biogénesis mitocondrial, y factores relacionados con la resistencia a la insulina como la inflamación y el estrés oxidativo.

Como describen los autores, el acúmulo de especies lipídicas (TG, DAG y LCACoA) en el músculo se acompaña de una menor actividad enzimática de M-CPT, enzima clave en el control de la oxidación mitocondrial de ácidos grasos, siendo éste un posible mecanismo involucrado en el desarrollo de lipotoxicidad. A su vez verificaron una disminución en la masa proteica de PPAR $\alpha$ que juega un rol importante en el control transcripcional de la oxida-

\section{BIBLIOGRAFÍA}

1. Cusi K. The role of adipose tissue and lipotoxicity in the pathogenesis of type 2 diabetes. Curr Diab Rep 2010; 10:306-315.

2. Ferrara D, Montecucco F, Dallegri F, Carbone F. Impact of different ectopic fat depots on cardiovascular and metabolic diseases. J Cell Physiol 2019; 1-12.

3. Consitt LA, Bell JA, Houmard JA. Intramuscular lipid metabolism, insulin action, and obesity. IUBMB. Life 2009; 61, 47-55.

4. Randle P, Garland P, Newsholme EA, Hales C. The glucose fatty acid cycle. Its role in insulin sensitivity and the metabolic disturbances of diabetes mellitus. Lancet 1963;1:785-789.

5. Metcalfe LK, Smith GC, Turner N. Defining lipid mediators of insulin resistance. Controversies and challenges. J Mol Endocrinol 2018; 62, R65-R82. ción de ácidos grasos al estimular la expresión de genes involucrados en la misma.

Los resultados del trabajo publicado suman conocimiento a un tema de crucial importancia para identificar objetivos potenciales para el desarrollo de nuevas drogas, a fin de reducir el riesgo de trastornos asociados a los lípidos y las enfermedades metabólicas y cardiovasculares. Claramente se necesitan más trabajos para entender cómo funcionan las redes lipídicas que existen entre órganos en la regulación de la homeostasis de los carbohidratos.

Dra. Gabriela Berg
Prof. Asociada de Bioquímica Clínica,
Facultad de Farmacia y Bioquímica (UBA)
Investigadora Independiente
en Salud del CONICET,
Laboratorio de Lípidos y Aterosclerosis,
Instituto de Fisiopatología y
Bioquímica Clínica, UBA

6. Miksztowicz V, Schreier L, McCoy M, Lucero D, Fassio E, Billheimer J, Rader DJ, Berg G. Role of SN1 lipases on plasma lipids in metabolic syndrome and obesity. ArteriosclerThromb Vasc Biol 2014 Mar; 34(3):669-75.

7. Barchuk M, Miksztowicz V, Zago V, Cevey A, López G, Goren N, Friedman S, Gelpi RJ, Morales C, Fernandez Tomé MDC, Schreier L, Berg G. Endothelial lipase is an alternative pathway for fatty acid release from lipoproteins: evidence from a high fat diet model of obesity in Rats. Lipids 2018 Oct; 53(10):993-1003.

8. Kiens B. Skeletal muscle lipid metabolism in exercise and insulin resistance. Physiol Rev 2006; 86(1): 205-43.

9. Bandet $\mathrm{CL}$, Tan-Chen S, Bourron O, Le Stunff H, Hajduch E. Sphingolipid metabolism: new insight into ceramideinduced lipotoxicity in muscle cells. Int J Mol Sci 2019 Jan 23; 20(3). 